\title{
Diagnostic Approach to Myocarditis Mimicking Myocardial Infarction at Initial Presentation
}

\author{
Craig Basmana, Pratik R. Agrawal ${ }^{\mathrm{a}}$, Chad McRee ${ }^{\mathrm{b}}$, Louis Saravolatz ${ }^{\mathrm{a}}$, \\ Carol Chen-Scarabellic ${ }^{c}$ Tiziano M. Scarabellia, b, c, d
}

\begin{abstract}
We present a case of a 35-year-old male patient with a 12-hour history of sudden-onset, crushing chest pain and associated complaints of profuse diaphoresis, nausea and vomiting. The patient was transferred to our institution from an outside hospital for evaluation and possible emergent catheterization. Left heart catheterization was conclusive for normal coronary arteries and a ventriculogram revealed a left ventricular ejection fraction of approximately $45 \%$. Due to a suspicion of myocarditis based on clinical history, pertinent serology tests were ordered, which were found to be negative. Cardiac magnetic resonance on delayed enhancement imaging showed typical sub-epicardial enhancement in a pattern most consistent with myocarditis. The patient was eventually diagnosed with myocarditis and discharged home later, without needing a myocardial biopsy. We present and discuss here the indications of myocardial biopsy and compare the relative utility of cardiac magnetic resonance imaging in formulating the diagnosis of myocarditis.
\end{abstract}

Keywords: Myocarditis; Cardiac MRI; Endomyocardial biopsy

\section{Introduction}

Myocarditis is an inflammatory process of the myocardium, which has variable manifestations. The most difficult aspect of this disease process is the diagnosis on initial presentation. Myocardial biopsy is the gold standard [1], and can play a crucial role in the diagnostic and prognostic evaluation of myocarditis. However, because of its low sensitivity and invasive

\footnotetext{
Manuscript accepted for publication July 21, 2016

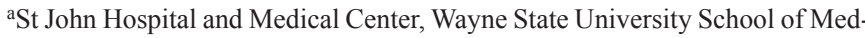
icine, Detroit, MI, USA

bUniversity of Alabama at Birmingham (UAB) Medical Center, Birmingham, Alabama, AL, USA

'Birmingham Veterans Affairs Medical Center, Birmingham, AL, USA

${ }^{\mathrm{d} C}$ Corresponding Author: Tiziano M. Scarabelli, Center for Heart \& Vessel Preclinical Studies; St John Hospital \& Medical Center, Wayne State University School of Medicine, Detroit, MI, USA. Email: tscarabelli@hotmail.com
}

doi: https://doi.org/10.14740/cr485w approach, it is only indicated in certain clinical scenarios [1]. Various other diagnostic approaches have been investigated, including the use of cardiac magnetic resonance imaging (MRI) solely and in conjunction with a myocardial biopsy. Evidence-based conclusions have shown great improvement in the outcomes of such protocols. This report discusses a case in which myocarditis had a mixed presentation, which led to a delayed diagnosis. Ultimately, the patient was spared a myocardial biopsy and was given a presumptive diagnosis of myocarditis based on the clinical picture and cardiac MRI.

\section{Case Report}

A 35-year-old male with no significant past medical history presented to our institution from an outside hospital with a 12-h history of sudden-onset crushing chest pain, described as $9 / 10$ in severity. The pain started at around 3:00 am, while the patient was lying in bed watching television. He stated that the pain radiated bilaterally and was associated with profuse diaphoresis, nausea and vomiting. He took two tablets of ibuprofen $(200 \mathrm{mg})$ with minor improvement and slept until the afternoon, when he was awakened by a new episode of severe chest pain and diaphoresis. He then presented to the emergency department.

In the emergency room, an electrocardiogram (EKG) documented ST segment elevation in lead I and aVL, and ST segment depression in lead III (Fig. 1). Preliminary lab work revealed a troponin $\mathrm{T}$ level of $25 \mathrm{ng} / \mathrm{mL}$. A chest X-ray was also obtained and showed mild cardiomegaly, in the absence of additional pathological findings (Fig. 2). The patient was therefore transferred to our institution for further evaluation and possible emergent catheterization.

Upon arrival to our hospital, repeat lab work showed a troponin level of $21.8 \mathrm{ng} / \mathrm{mL}$, CK-MB of $77 \mathrm{ng} / \mathrm{mL}$, creatinine of $0.6 \mathrm{mg} / \mathrm{dL}, \mathrm{Hb}$ of $12.6 \mathrm{~g} / \mathrm{dL}, \mathrm{WBC}$ count of $7,300 / \mu \mathrm{L}$ and a C-reactive protein level of $131 \mathrm{mg} / \mathrm{L}$. The patient was taken for an emergent left heart catheterization, which was conclusive for normal coronary arteries. A ventriculogram was also performed and revealed a left ventricular ejection fraction (LVEF) of approximately $45 \%$ with mild hypokinesis of the antero-apical and infero-apical walls. Consequently, the patient was admitted to the coronary care unit (CCU) for further management.

On arrival to the CCU, the patient's vitals were found to be unremarkable. The patient was afebrile with blood pressure 


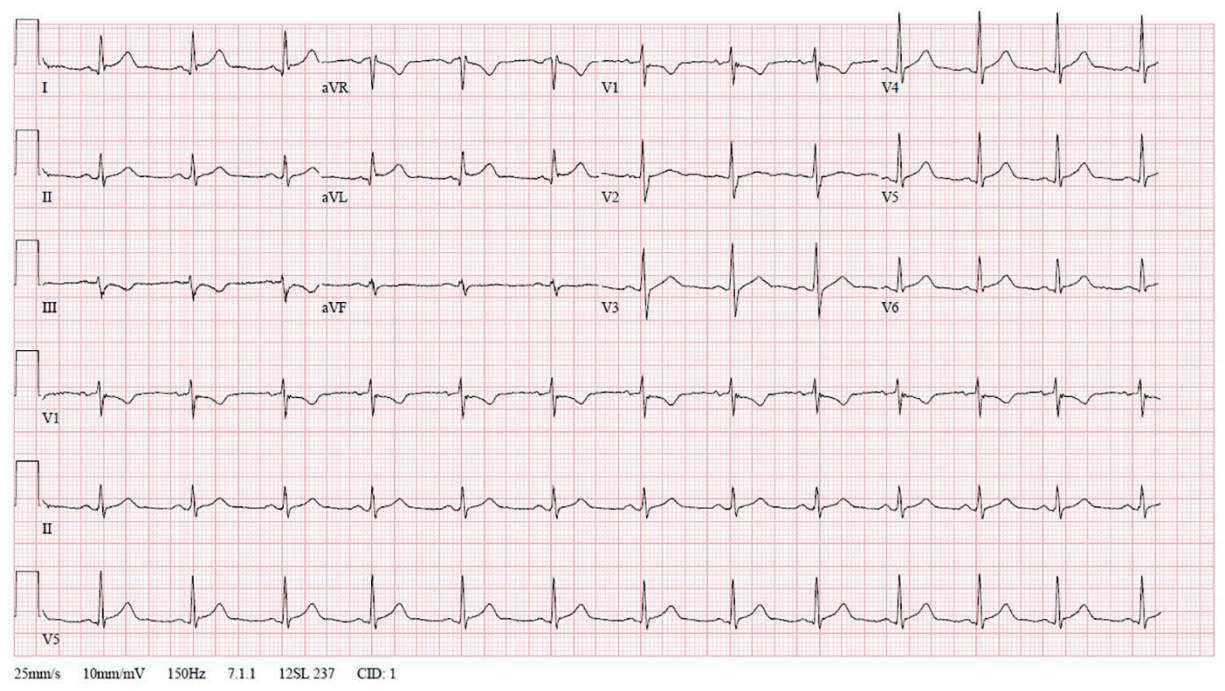

Figure 1. Electrocardiogram of presenting patient acquired upon arrival in the emergency department. Illustrative of ST elevation in lead I and $\mathrm{aVL}$, and ST depression in lead III.

measurement of $117 / 72 \mathrm{~mm} \mathrm{Hg}$, pulse 77 beats/min, respiratory rate 20 breaths/min and an oxygen saturation of $100 \%$. Patient's height and weight were measured 65 inches and 187 pounds, respectively. The patient's physical examination was largely unremarkable. More specifically, the patient did not show any signs of jugular venous distention, thyromegaly, or a carotid bruit. Cardiac auscultation revealed a regularly regular rhythm of the heart, with a normal S1/S2 and void of extra cardiac sounds and rubs, murmurs or thrills. There was no chest wall tenderness. Lungs were clear to auscultation bilaterally and no rales, rhonchi or wheezing could be appreciated. The abdomen was soft to palpation, non-tender, non-distended, with no evidence of guarding or rebound pain. Upon examination of the extremities, the patient did not show any evidence of dependent edema.

Social history was significant for a 5 pack-year history of smoking cigarettes, although the patient had quit smoking 5 years prior to this presentation. He denied any use of alcohol or illicit drugs and his family history was non-contributory. He also denied any prior medical condition, the use of any overthe-counter and/or prescribed medications.

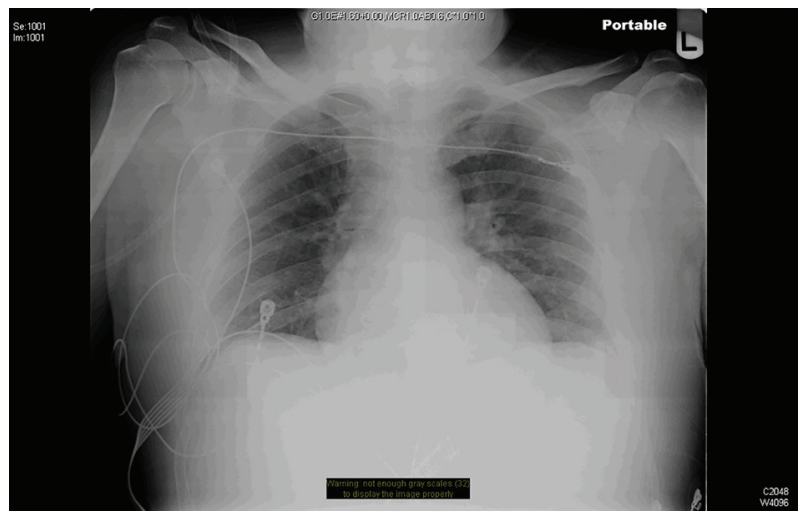

Figure 2. Chest X-ray obtained on arrival in the emergency department.
Further questioning in the CCU disclosed that the patient had experienced a fever and profuse sweating in the absence of any other symptoms 4 days prior to the onset of the presenting chest pain. Due to the high suspicion of myocarditis, a cardiac MRI and pertinent serology tests were ordered. Cardiotropic viruses including parvovirus B19, human herpes virus 6 , human immunodeficiency virus-1, hepatitis B virus, EpsteinBarr virus, cytomegalovirus, influenza virus $B$, adenovirus and Coxsackie virus were not detected in the peripheral blood. Borrelia, rickettsia and legionella serology were also negative. Blood cultures did not yield bacteria or fungal growth.

Cardiac MRI furnished evidence that the left and right ventricles were both normal in size and in systolic function. Likewise, there was no evidence of segmental wall motion abnormalities for either ventricle. T2-weighted imaging showed no evidence of myocardial edema or intra-myocardial iron deposition. However, on delayed enhancement imaging, there was sub-epicardial enhancement in the basal to mid inferior, inferolateral and anterolateral segments, as well as in the apical inferior and lateral segments in a pattern which was most consistent with myocarditis (Fig. 3).

\section{Outcome and follow-up}

The patient was discharged with the diagnosis of idiopathic myocarditis, and was advised bed rest along with supplemental home oxygen.

\section{Discussion}

Myocarditis can present with a variable clinical picture and diagnosis at initial presentation can often be a challenge. Myocarditis may mimic myocardial ischemia both clinically and electrocardiographically, which is particularly evident in younger patients [2]. Additionally, there are many instances in 


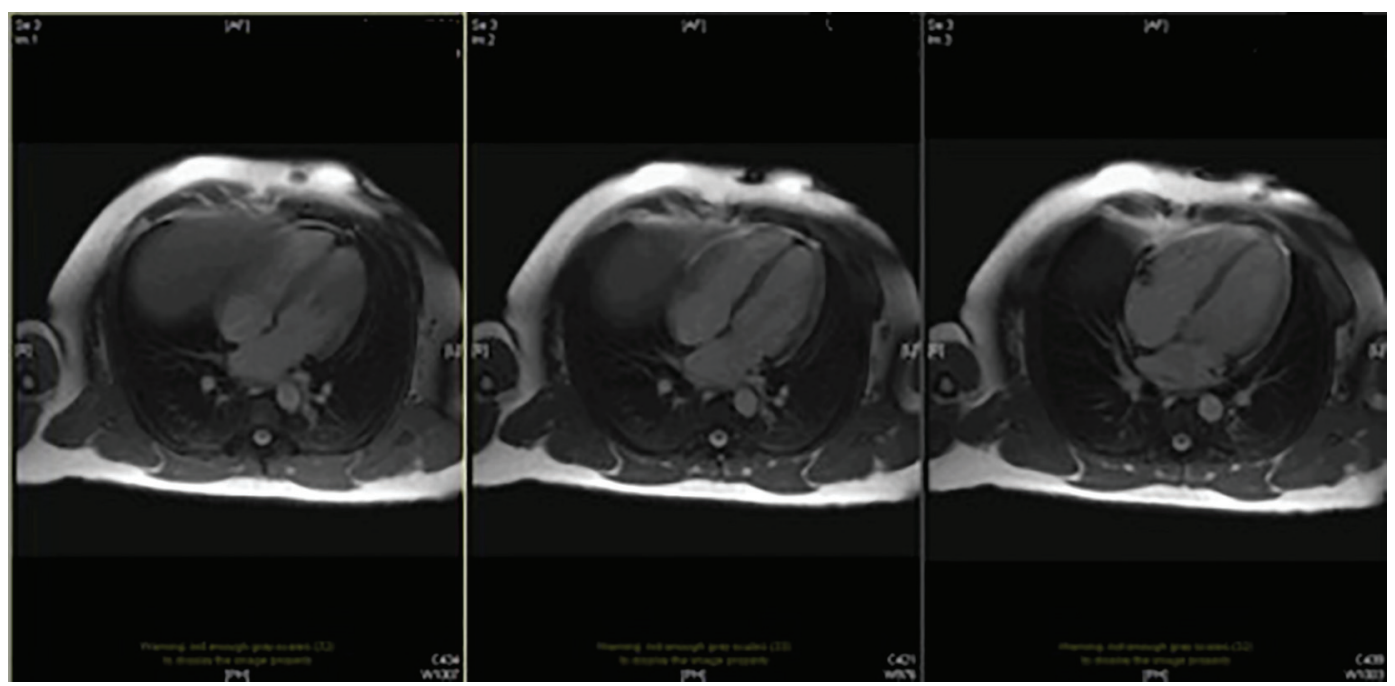

Figure 3. Cardiac MRI of adenosine stress with contrast. MRI results show indication of subepicardial scarring suggesting myocarditis.

which symptomatic cases of myocarditis present with a syndrome of heart failure or dilated cardiomyopathy [3]. Chest pain is typically associated with concomitant pericarditis. Myocarditis may also present with unexpected sudden death, presumably due to ventricular arrhythmias.

Acute myocarditis should be suspected whenever a patient, especially a younger patient, presents with new onset of cardiac abnormalities otherwise unexplained, such as heart failure, cardiac arrhythmias, or conduction disturbances [2]. A history of recent upper respiratory infection or enteritis may also be elicited in the majority of viral myocarditis cases [4].

Furthermore, there are several other settings in which the diagnosis of myocarditis should be considered. Firstly, in cases where systemic infections (viral, bacterial, rickettsial, fungal or parasitic) are associated with new abnormalities in cardiovascular function. Since many cardiotropic viruses including Coxsackie A are also myotropic, the concurrent presence of myalgia should enhance the suspicion of myocarditis [5]. Secondly, cases in which acute viral infections are accompanied by tachycardia out of proportion to fever. Myocarditis should also be considered when a patient, especially a young patient, presents with clinical signs and symptoms of an acute myocardial infarction with a normal coronary angiogram. And lastly, cases in which a patient develops symptoms of heart failure associated with rash and eosinophilia following a new drug or vaccine.

\section{Diagnostic challenge of myocarditis}

The diagnosis of myocarditis is particularly difficult because an established non-invasive gold standard does not exist. Furthermore, sensitivity of the current invasive gold standard which is endomyocardial biopsy (EMB) [1] is very low due to the variability in interpretation and sampling error [6]. Another diagnostic mean, cardiac MRI, is useful to diagnose epicardial and mid wall inflammation and edema in patients who would have a low yield from right ventricular EMB [7]. Therefore, al- though histology remains the gold standard, low-risk patients are often given a presumptive diagnosis of myocarditis, if the clinical presentation and imaging studies are suggestive. As shown in a recent similar case report by Zhang et al, EKG, coronary angiography and cardiac MRI were used in combination for the diagnosis of viral myocarditis without a myocardial biopsy [8].

\section{EKG manifestations of myocarditis}

The EKG findings in myocarditis vary considerably. Sinus tachycardia and non-specific T wave and ST segment changes are most common. Occasionally, the changes may include ST-segment elevation or depression, and pathologic Q waves [9]. Rhythmic disturbances, such as supraventricular and ventricular arrhythmias or atrial and ventricular conduction delays, may appear secondary to myocardial inflammation. High grade heart block is infrequent, and is more common in Lyme $\square \mathrm{s}$ disease, cardiac sarcoidosis and idiopathic giant cell myocarditis [10].

\section{Utility of viral serological tests}

Virus serology for cardiotropic viruses such as Coxsackie virus is commonly used in clinical practice to aid in the diagnosis of myocarditis. However, such serological tests are known to have poor sensitivity, and evidence regarding their clinical utility is lacking $[1,11]$. A negative viral serological profile does not rule out a diagnosis of myocarditis. In a recent study of 124 patients with suspected myocarditis, viral serology was found to have no relevance in the diagnosis of viral myocarditis [11]. Detection of acute viral infection via serological tests was shown to have no co-relation with viral genome detection in EMB in the study patients. Furthermore, routine detection for viral genome in EMB has not been recommended in inter- 
Table 1. The 2007 AHA/ACCF/ESC Guidelines for Endomyocardial Biopsy (Modified From the AHA/ACCF/ESC "Role of Endomyocardial Biopsy in the Management of Cardiovascular Disease" [1])

\section{Class I: clinical scenarios where EMB "should be performed"}

1 New-onset heart failure (HF) with onset $<2$ weeks duration, in addition to: 1) normal or dilated left ventricle (LV); 2) hemodynamic compromise

2 New onset HF with duration 2 weeks to 3 months, in addition to either dilated LV and new ventricular arrhythmias or second/third degree heart block, or failure to respond to usual care within 1 to 2 weeks

Class IIa: clinical scenarios where EMB "may be considered reasonable"

3 HF of more than 3 months duration, in addition to either dilated LV and new ventricular arrhythmias or second/third degree heart block, or failure to respond to usual care within 1 - 2 weeks

$4 \quad$ HF with dilated cardiomyopathy of any duration with suspected allergic reaction and/or eosinophilia

5 HF with suspected anthracycline cardiomyopathy

6 HF with unexplained restrictive cardiomyopathy

7 Suspected cardiac tumors (exception of typical myxomas)

8 Unexplained cardiomyopathy in the pediatric population

Class IIb: clinical scenarios where EMB "may be considered"

9 HF with duration of 2 weeks to 3 months with a dilated LV, without new arrhythmia/heart block, that does respond to usual care within 1 - 2 weeks

10 Suspicion for iron overload in unexplained HF of $>3$ months duration with a dilated LV, without arrhythmias/heart block, that does respond to usual care

11 HF associated with unexplained hypertrophic cardiomyopathy (if an infiltrative or storage disease is suspected)

12 Suspected arrhythmogenic right ventricular cardiomyopathy when other evaluations have been inconclusive

13 Unexplained ventricular arrhythmia

national guidelines [1].

\section{Role of cardiac MRI in myocarditis diagnosis}

Use of cardiac MRI is growing in the diagnosis of myocarditis, and some studies show that it may help with prognostication as well. There are three pertinent characteristics of cardiac MRI that propel it to the forefront diagnostic tool of myocarditis. Firstly, cardiac MRI allows for the examiner to assess cardiac function indirectly [12]. There is an inverse relationship between the degree of inflammation present and the residual cardiac function. This is supported by one study in particular, which assessed 19 patients, who presented with a strong pretest probability for myocarditis. The results showed there was an observable association between myocardial involvement on cardiac MRI and the cardiac function [13].

Another factor in support of the role of MRI for diagnosis of myocarditis is the help provided by cardiac MRI in directing myocardial biopsy. The sensitivity of myocardial biopsy is largely limited by its off-target sampling. There is evidencebased support that myocardial biopsy's yield improves significantly in conjunction with cardiac MRI. This is exemplified in one study of 32 patients with a substantial probability of myocarditis that underwent gadolinium-enhanced cardiac MRI prior to myocardial biopsy. Biopsy was taken in both the regions of foci detected on cardiac MRI and outside the regions. It was concluded that 19 out of the 21 patients that underwent myocardial biopsy in the active regions were found to have active myocarditis present on biopsy. Yet, when myocardial biopsy was performed outside the image detected regions, a conclusive diagnosis of active myocarditis was reached in only one out of 11 patients [7].

Lastly, cardiac MRI can help differentiate myocarditis from ischemic cardiomyopathy, and can also aid in diagnosing the culprit virus. For example, patients who present with a case of myocarditis have characteristic epicardial and myocardial involvement, whereas ischemic cardiomyopathy patients tend to present with involvement of the endocardium. The use of cardiac MRI can further allow one to differentiate between several causes of viral pathogens responsible for myocarditis. An example would be that patients with herpes virus 6 present with a characteristic septal foci enhancement, while patients with parvovirus B19 depict foci in the areas of the subepicardium of the lateral left ventricle [14].

Apart from its diagnostic yield, cardiac MRI may also aid in prognostication of myocarditis. In a small study with 3-year follow-up, myocarditis patients had repeat cardiac MRI at 4 weeks after clinical onset of the disease. Contrast enhancement ratio at 4 weeks was predictive of the functional and clinical long-term outcome [15].

\section{When should EMB be performed?}

There are no randomized controlled treatment data advising on the utility of EMB. Hence, recommendations regarding its use are based on case-control series and expert opinion. With 
the exception of monitoring for cardiac allograft rejection, the 2007 AHA/ACCF/ESC statement recommended two clinical settings in which EMB should be performed, one being in the event of an unexplained new-onset heart failure (HF) of less than 2 weeks' duration associated with a normal-sized or dilated left ventricle in addition to hemodynamic compromise (clinical scenario 1) and the other event being an unexplained new-onset HF of 2 weeks to 3 months' duration associated with a dilated left ventricle and new arrhythmias such as ventricular arrhythmias, Mobitz type II second-degree atrioventricular (AV) block, third-degree AV block, or failure to respond to usual care within 1 - 2 weeks (clinical scenario 2) [1]. Additional recommendations and clinical scenarios are described in Table 1 .

\section{Conclusion}

The patient in this case report was discharged home with a diagnosis of myocarditis without needing EMB. As myocarditis may have a varied clinical presentation, its diagnosis is often challenging. The differential for chest pain and ST segment elevation is broad. Though myocarditis is a less frequent etiology, there are several clinical scenarios where practitioners should be aware of the possibility. The utility of cardiac MRI is expanding as a non-invasive diagnostic tool for myocarditis. Evidence-based medicine has shown a growing role for cardiac MRI within the realm of diagnosis and a continuing need for it even without EMB, the current "gold standard". The benefits of cardiac MRI include prognostication, augmenting yield of an EMB and diagnosis without EMB.

\section{Learning points}

1) Myocarditis can have mixed clinical manifestations and should be kept in mind as a possibility in cases presenting with signs and symptoms of myocardial infarction but with normal coronary angiography, as discussed in the present case report

2) Low sensitivity, invasive approach and a relatively higher rate of complication limit the use of EMB for diagnosis of myocarditis.

3) Cardiac MRI offers a non-invasive, reliable tool for diagnosis of myocarditis; additional advantages include repeatability factor, indirect assessment of relative cardiac function and prognostication.

\section{Conflicts of Interest}

The authors have no conflicts of interest to report.

\section{References}

1. Cooper LT, Baughman KL, Feldman AM, Frustaci A, Jessup M, Kuhl U, Levine GN, et al. The role of endomyocardial biopsy in the management of cardiovas- cular disease: a scientific statement from the American Heart Association, the American College of Cardiology, and the European Society of Cardiology. Circulation. 2007;116(19):2216-2233.

2. Dec GW, Jr., Waldman H, Southern J, Fallon JT, Hutter AM, Jr., Palacios I. Viral myocarditis mimicking acute myocardial infarction. J Am Coll Cardiol. 1992;20(1):8589.

3. D'Ambrosio A, Patti G, Manzoli A, Sinagra G, Di Lenarda A, Silvestri F, Di Sciascio G. The fate of acute myocarditis between spontaneous improvement and evolution to dilated cardiomyopathy: a review. Heart. 2001;85(5):499504.

4. Ramazzina C, Bremerich J, Linka A, Eriksson U. [Asymptomatic myocarditis after infection of the upper respiratory tract]. Dtsch Med Wochenschr. 2005;130(21):13111313.

5. Magnani JW, Dec GW. Myocarditis: current trends in diagnosis and treatment. Circulation. 2006;113(6):876-890.

6. O'Connell JB, Henkin RE, Robinson JA, Subramanian R, Scanlon PJ, Gunnar RM. Gallium-67 imaging in patients with dilated cardiomyopathy and biopsy-proven myocarditis. Circulation. 1984;70(1):58-62.

7. Mahrholdt H, Goedecke C, Wagner A, Meinhardt G, Athanasiadis A, Vogelsberg H, Fritz P, et al. Cardiovascular magnetic resonance assessment of human myocarditis: a comparison to histology and molecular pathology. Circulation. 2004;109(10):1250-1258.

8. Zhang J, He S, Qi X, Li Y. Combined electrocardiography, coronary angiography and magnetic resonance imaging for the diagnosis of viral myocarditis: A case report. Exp Ther Med. 2014;7(6):1643-1646.

9. Wang K, Asinger RW, Marriott HJ. ST-segment elevation in conditions other than acute myocardial infarction. $\mathrm{N}$ Engl J Med. 2003;349(22):2128-2135.

10. Nunes H, Freynet O, Naggara N, Soussan M, Weinman P, Diebold B, Brillet PY, et al. Cardiac sarcoidosis. Semin Respir Crit Care Med. 2010;31(4):428-441.

11. Mahfoud F, Gartner B, Kindermann M, Ukena C, Gadomski K, Klingel K, Kandolf R, et al. Virus serology in patients with suspected myocarditis: utility or futility? Eur Heart J. 2011;32(7):897-903.

12. Olimulder MA, van Es J, Galjee MA. The importance of cardiac MRI as a diagnostic tool in viral myocarditisinduced cardiomyopathy. Neth Heart J. 2009;17(12):481486.

13. Friedrich MG, Strohm O, Schulz-Menger J, Marciniak H, Luft FC, Dietz R. Contrast media-enhanced magnetic resonance imaging visualizes myocardial changes in the course of viral myocarditis. Circulation. 1998;97(18):1802-1809.

14. Mahrholdt H, Wagner A, Deluigi CC, Kispert E, Hager S, Meinhardt G, Vogelsberg H, et al. Presentation, patterns of myocardial damage, and clinical course of viral myocarditis. Circulation. 2006;114(15):1581-1590.

15. Wagner A, Schulz-Menger J, Dietz R, Friedrich MG. Long-term follow-up of patients paragraph sign with acute myocarditis by magnetic paragraph sign resonance imaging. MAGMA. 2003;16(1):17-20. 